\title{
Factores psicosociales asociados a la ideación suicida y el parasuicidio en adolescentes
}

Psychosocial factors associated to suicidal ideation and parasuicidal behavior in adolescents

\author{
Blanca S. Fraijo Sing \\ Nehemías Cuamba Osorio \\ Víctor Corral Verdugo \\ César Tapia Fonllem \\ Martha Montiel Carbajal \\ Universidad de Sonora
}

\begin{abstract}
Resumen
El objetivo del presente trabajo fue probar un modelo psicosocial explicativo del comportamiento suicida (estudiado como ideación suicida y conducta parasuicida) en adolescentes. Se utilizó una muestra de 102 adolescentes separados en dos grupos, un grupo con 51 adolescentes identificados con comportamiento suicida que recibían atención en un centro psicoterapéutico de adolescentes; y uno control del mismo número de participantes de la población de Hermosillo, Sonora. Se les aplicó una batería de pruebas para medir factores sociales y psicológicos, obteniendo también variables sociodemográficas. Se probó un modelo de ecuaciones estructurales donde la variable dependiente fue el comportamiento suicida, y las independientes los factores sociales y psicológicos. En los resultados se identificaron 7 factores, 3 de ellos (el maltrato infantil, la comunicación familiar y el rechazo parental) afectaron de forma indirecta a la variable dependiente, a través de un agregado de variables psicológicas individuales que incluía depresión, ansiedad, baja autoestima y el déficit en habilidades de afrontamiento. Este agregado afectó a la variable dependiente sólo en su faceta de ideación suicida, la cual influyó en las conductas parasuicidas. Se concluye que el presente trabajo ofrece una aproximación a la búsqueda de explicaciones al fenómeno de la conducta suicida. Aunque las explicaciones por necesidad deben incluir factores multi-causales, en nuestro modelo los factores familiares e individuales se presentan como los más importantes. Se discuten las posibles implicaciones para el trabajo preventivo de este fenómeno en adolescentes.
\end{abstract}

Palabras clave: Ideación Suicida, Parasuicidio, Adolescencia, Disfunción Familiar, Disfunción Individual.

Nota del Autor:

Blanca S. Fraijo Sing, Departamento de Psicología, Universidad de Sonora; Nehemías Cuamba Osorio, Departamento de Psicología, Universidad de Sonora, Unidad Regional Norte; Víctor Corral Verdugo, Departamento de Psicología, Universidad de Sonora; César Tapia Fonllem, Departamento de Psicología, Universidad de Sonora; Martha Montiel Carbajal, Departamento de Psicología, Universidad de Sonora.

La correspondencia en relación con este artículo debe dirigirse a Blanca S. Fraijo Sing, Departamento de Psicología, Universidad de Sonora, Blvd. Encinas y Rosales, s/n, Col. Centro, C.P. 83000, Hermosillo, Sonora, México.

Correo electrónico: bfraijo @ sociales.uson.mx 


\section{Abstract}

This study was aimed at testing an explanatory psicosocial model of suicidal behavior (indicated by suicidal ideation and parasuicidal conduct) in teenagers. The sample was formed by one hundred and two teenagers divided into two groups, one with fifty-one teenagers that had engaged in suicidal behavior and who were currently attending at a psicotherapeutic center. A group was formed with the same number of subjects from Hermosillo, Sonora, Mexico. A battery of instruments was administered to assess social and psychological factors as well as sociodemographic variables. A structural equation model was specified and tested including suicidal behavior as dependent variable, and social and psychological factors as independent ones. Seven factors were identified; three of them (child abuse, family communication, and parental rejection) affected the dependent variable indirectly, through a set of individual psychological variables that included depression, anxiety, low self-esteem, and lack of coping skills. This set affected the dependent variable only in its suicidal ideation facet, which in turn influenced parasuicidal conduct. These findings offer an approach to the search of the determinants of suicidal behavior. Although these determinants include multicausal factors, in our model, individual and family factors are presented as the most important ones. The implications for possible preventive strategies regarding this phenomenon are also addressed

Keywords: Suicidal Ideation, Parasuicidal conduct, Teenagers, Family Dysfunction, Individual Dysfunction.

El comportamiento suicida es un fenómeno multifactorial con tendencia epidemiológica mundial ascendente, y considerado como un problema relevante de salud pública (Gunnell, 2002). La OMS (2009) estimó que en el año 2000, cerca de 1 millón de personas murieron por esta causa, de las cuales 100,000 fueron adolescentes y se consideró que, de continuar esta tendencia, más de 1.5 millones de personas al año morirán por este problema (Bertolote \& Fleischmann, 2002).

Desjarlais Eisenberg, Byron y Kleinman (1995) plantean que el suicidio se encuentra entre las primeras causas de muerte en los países que mantienen su registro estadístico. Es necesario considerar el sub-registro, debido a las dificultades legales y éticas que conlleva el registro oficial y que éste sólo se enfoca en el suicidio consumado, dejando de lado otros tipos de comportamiento suicida (González-Forteza, Mariño, Rojas, Mondragón, \& Medina-Mora, 1998).

En niños y adolescentes existe una tendencia a desvalorizar su ocurrencia, lo cual se traduce en registros estadísticos que no reflejan la magnitud real del problema (Mardomingo, 1998; Montenegro \& Guajardo, 2000; Marquet, 2008).
Pese a esto, se ha observado un incremento en la prevalencia del fenómeno en esta etapa de la vida (OPS, 1998; Larraguibel, Martínez, Valenzuela, González, \& Schiattino, 2001) y se puede considerar como un problema de salud pública debido a la alta demanda de recursos profesionales y financieros que representa para los sistemas de salud (Huey, Henggeler, \& Rowland, 2004; Palacios, Barrera, Ordoñez, \& Peña, 2007). La ausencia de criterios estandarizados para recolectar la información y de programas de vigilancia epidemiológica sobre el suicidio hacen más difícil su conocimiento y registro (González-Forteza et al, 1998), lo cual incide de forma negativa en su prevención y tratamiento, lo mismo que en la capacidad para medir su impacto en la sociedad.

\section{Comportamiento Suicida}

En 1897, Durkheim realizó la primera investigación sociológica de esta situación; definió al suicidio en términos de un acto personal, propositivo y consciente, mediante el cual el sujeto que lo realiza busca terminar con su vida (Durkheim, 1897). A partir de esta conceptuación se generó un cambio en la perspectiva del evento, se le otorgó importancia a los elementos 
contextuales que rodean al individuo y se comenzaron a atender los motivos subyacentes. Con esto, se sentaron las bases para que el problema fuera abordado e investigado desde distintos enfoques, considerándolo como un fenómeno complejo que incluye factores neurobiológicos (Guiao \& Esparza, 1995; Harris \& Lennings, 1995), familiares, (Steinhaussen \& Winckler, 2004; Cubillas et al., 2006), genéticos (Stefulj et al., 2004), psicológicos (Andrade, Betancourt, \& Camacho Valladares, 2003), sociales (Durkheim,1897), culturales (Leslie, Stein, \& Rotheram-Borus, 2002) y otros aún en estudio.

Además se observó que estos comportamientos tienen un curso más o menos definido, con diferentes instancias y categorías de análisis; abarcan conportamientos que van desde la ideación suicida a las conductas auto-lesivas, pasando por el intento suicida y terminando en el acto consumado (Fernández, 2002).

Es necesario hacer una diferencia entre el comportamiento suicida y el suicidio consumado. Para la OMS (2009) el comportamiento suicida es toda acción con la que el individuo se causa daño a sí mismo, independientemente del grado de letalidad del acto y de que se conozcan o no los motivos subyacentes; por lo que, cuando se hace referencia al comportamiento suicida, no sólo se trata de la conducta consumada, sino también forman parte: el intento suicida, la ideación suicida y las conductas auto-lesivas (Durkheim, 1897; Diekstra 1985; Arlaes, Hernández, Álvarez, \& Cañizarez, 1997).

Casullo (2002) afirma que este problema abarca conductas que incluyen, desde gestos e intentos manipuladores hasta intentos fallidos de terminar con la propia vida, haciendo referencia a comportamientos deliberados sin intención de finalizar en la muerte pero que provocan daños en el sujeto. El suicidio consumado no es más que un elemento de todo un proceso; la culminación de una serie de comportamientos encadenados, llevados a cabo en función de la ideación de la persona y de su intencionalidad suicida (Bobes, 2004).
Otro aspecto importante de resaltar, al momento de definir el comportamiento suicida, es su independencia frente al objetivo de conseguir la muerte, lo cual quiere decir que no importa si el individuo consigue quitarse la vida o no, ni tampoco la letalidad del método utilizado para realizarlo. Castañeda (2003) afirma que en ocasiones la conducta suicida no tiene como objetivo causar la muerte, sino más bien se constituye en un medio de comunicación o expresión de emociones o sentimientos como rabia, cólera o frustración ante situaciones conflictivas. En otras instancias los objetivos son llamar la atención, acabar con el sufrimiento o vengarse de alguna figura de autoridad (González-Forteza et al, 2002).

De igual forma, en los comportamientos suicidas es necesario diferenciar aquellas conductas mortales de las no mortales, de acuerdo con el resultado de las mismas (OPS, 1998). Las conductas suicidas no mortales han sido llamadas parasuicidas e implican comportamientos aparentemente dirigidos a quitarse la vida, pero que se encuentran motivados por una intención manipuladora, o en ocasiones de placer. Pese a ello, estas conductas pueden causar daños significativos en el ejecutante.

Mack (1986), tratando de explicar este fenómeno, desarrolla un modelo con base en el estudio del suicidio consumado en jóvenes, el cual llamó "Modelo Arquitectónico" y consta de varios elementos:

1. Macrocosmos: la influencia que ejercen la cultura, la actividad económica, los factores sociopolíticos y el sistema educativo.

2. Vulnerabilidad biológica y los factores genéticos.

3. Experiencias tempranas que tienen influencia sobre etapas iniciales del desarrollo. En ellas la familia tiene un peso predominante.

4. La organización de la personalidad y la autoestima. 
5. Las relaciones del individuo, que incluye su relación con los padres, su aceptación o rechazo, el grado de separación, identificaciones, lazos con otros adultos y relaciones con los amigos.

6. La psicopatología, que establece la existencia de cuadros depresivos y alcoholismo.

7. La ontogenia en la relación existente entre el desarrollo y la muerte.

8. Las circunstancias vitales como factores precipitantes, tanto biológicos como socio-familiares.

\section{Factores de riesgo implícitos}

Frente a los factores de riesgo implícitos, Arlaes, Hernández, Alvarez y Cañizarez (1997) afirman que el suicidio, como hecho de causa multifactorial (biológica, psicológica, social y la interacción entre variables), evidencia las fallas en el mecanismo de adaptación del sujeto. Para Fernández (2002), los factores de riesgo constituyen situaciones, condiciones o eventos que en conjunto pueden aumentar la probabilidad de que una persona emita conductas voluntarias y conscientes dirigidas a causarse la muerte. La identificación y análisis de esos factores permite predecir hasta cierto punto los posibles comportamientos suicidas de un individuo, lo cual es básico para el diseño e instrumentación de estrategias de intervención.

Se puede establecer que el comportamiento suicida no es una conducta aleatoria y, desde sus primeras descripciones científicas, se observó la posibilidad de predecirlo. Por lo tanto, conocer los factores implícitos es de gran importancia para evaluar adecuadamente el riesgo en una persona. Es importante resaltar la naturaleza de los factores individuales frente a la presentación de la conducta suicida; por lo que, para algunas personas, un factor determinado es un elemento de riesgo; mientras que, para otros, puede no representar problema alguno (García de Jalón \& Peralta, 2002).
Nrugham, Larsson y Sund (2008) realizaron un estudio longitudinal con estudiantes noruegos cuya media de edad fue de 13.7 años y que padecían niveles altos de depresión; se hizo seguimiento al año y 5 años respectivamente. Los resultados mostraron que esta patología, en edades tempranas, es predictora de comportamientos suicidas entre los 15 y 20 años.

Reinherz, Tanner, Berger, Beardslee y Fitzmaurice (2006) investigaron en una población caucásica la presencia de estos comportamientos; destacaron una fuerte relación entre ideación suicida a los 15 años y mayores tasas de depresión, ideación suicida y problemas de comportamiento, e ideación suicida y de capacidades bajas de afrontamiento. Los jóvenes con ideación suicida a la edad de 15 años presentaron menor nivel de autoestima, menor capacidad para hacer frente a situaciones difíciles y mayor necesidad de apoyo social. Sean, Baser, Breeden, Neighbors y Jackson (2006) observaron estos comportamientos en la población afroamericana joven e informaron una prevalencia de $11.7 \%$ para la ideación suicida. De este porcentaje, el 34.6\% había realizado un plan y el $21 \%$ llevó a cabo un intento no planificado.

Hardt et al. (2008) en Canadá, observaron la presencia de factores como la separación de padres, la violencia doméstica en la infancia, como predictores de la presencia de conductas suicidas en una muestra de 575 adolescentes. En un estudio longitudinal hecho en Grecia, en el periodo 1998 a 2008, Vougiouklakis, Tsiligianni y Vassiliki (2009) trabajaron en individuos menores de 24 años; encontraron que en ese país la tasa de comportamientos suicidas era menor al de la media resto de Europa; llegaron a la conclusión de que el estilo de vida en Grecia es más tradicional y las familias presentan mayor cohesión que en el resto del continente. Otro estudio realizado en Suecia, encontró que las características de la familia del adolescente es un factor tanto de riesgo como protección para la presencia de estos comportamientos (Mittendorfer-Rutz, Wasserman, \& Rasmussen, 2008). 
En Corea del Sur, se identificaron 5 factores de riesgo en la presencia de comportamiento suicida en adolescentes de 13 a 18 años; entre los que se encontraban la pobre relación con los padres y la depresión en los padres, además de encontrar una menor tasa de intentos de suicidio en adolescentes que pertenecen a grupos de delincuencia o pandillas a razón de grupos de estudiantes que no pertenecen a estos grupos (Soo Kim \& Sil Kim, 2008).

Compton, Thompson y Kaslow (2005) encontraron en una muestra de afroamericanos una relación entre la disfunción familiar, la depresión y la presencia de comportamientos suicidas. Gónzalez - Forteza et al. (2002) encontraron que la prevalencia en la ideación suicida está relacionada a la mala comunicación entre padres e hijos; a su vez, otro factor presente es el bajo nivel de autoestima y estrategias de afrontamiento agresivas. Con un mayor riesgo de presencia de comportamiento en adolescentes impulsivos.

Barcelata, Duran y Lucio (2004) encontraron factores como el nivel de autoestima del adolescente y su relación con los padres como factores de riesgo, encontrando mayor prevalencia en el género masculino que en el femenino. Andrade, Betancourt y Camacho Valladares (2003) y Palacios, Barrera, Ordoñez y Peña (2007) manejaron factores más allá de la depresión, como el desamparo aprendido, procesos de duelo no resueltos y una falta de habilidades de afrontamiento como factores de riesgo; además, algunas investigaciones relacionadas con factores de tipo escolar (Palacios et al., 2007).

Valero (2008) señala un aumento preocupante en México de intentos de suicidio y suicidios en niños y adolescentes, expresando que en cerca del $49 \%$ de los casos se desconoce la causa. Asimismo, relaciona perturbaciones emocionales, tales como la depresión y ansiedad, con este fenómeno.

En relación a la depresión y ansiedad es importante destacar que la co-morbilidad de ambos trastornos multiplica el riesgo (Gutiérrez \& Contreras, 2006). Sauceda-García, Lara-Muñoz y
Focil-Marquez (2006) hicieron una comparación entre los factores de riesgo depresión e impulsividad, encontrando que la depresión es un factor de riesgo mayor que la impulsividad; además, los problemas familiares también estuvieron presentes. Rivera y Andrade (2006) trataron de identificar factores de protección en los adolescentes y encontraron el manejo de las emociones y el apoyo grupal como principales factores de protección.

Durkheim (1897) afirmó que este tipo de comportamiento refleja la relación de la persona consigo misma y con su comunidad. Por lo tanto, para entender las causas individuales del suicidio, se recurre al contexto social del individuo, el cual influye en sus actitudes con relación a su concepto de vida y muerte. Uno de los contextos sociales determinantes es el de la dinámica familiar y éste puede incluir el rechazo parental, los problemas de comunicación y la presencia de maltrato infantil, como factores influyentes (Monge, Cubillas, Román, \& Abril, 2007) que producen repercusiones en el plano individual o psicológico (Dwayri, 2011).

Por lo anterior, se observa que se han realizado distintos estudios, tanto a nivel internacional como en el país, para conocer su prevalencia, factores de riesgo y variables asociadas; sin embargo, se requiere de investigación sistemática en todos los ámbitos para mejorar el conocimiento del fenómeno y para instrumentar estrategias eficaces de prevención (Ruiz, Navarro, Torrente, \& Rodríguez González, 2005). Por otra parte, la mayor parte de los datos epidemiológicos frecuentemente derivan de encuestas retrospectivas, que consisten en preguntar al sujeto si ha cometido determinado comportamiento suicida, lo cual contiene un sesgo de memoria y el factor disposición del encuestado a proveer la información. Aun así, las investigaciones realizadas permiten contar con un perfil del adolescente con este tipo de conductas (González-Forteza et al., 2002). Lo anterior, hace necesario dar un paso adicional para determinar los factores psicosociales de riesgo del adolescente, partiendo de la identificación y evaluación en una población que ya cuenta con antecedentes de este tipo de comportamientos, la cual es atendida en su problemática, para 
generar un modelo explicativo de la conducta suicida en adolescentes. De tal forma, que se podrían generar estrategias preventivas y de intervención, no sólo considerando a los adolescentes, sino también a los niños. Por lo anteriormente expuesto, el objetivo del presente estudio fue generar un modelo explicativo del comportamiento suicida a partir de una muestra que presenta conductas relacionadas con el mismo.

\section{Método}

\section{Participantes}

La muestra estudiada está compuesta por 102 adolescentes divididos en dos grupos, de igual número de participantes $(n=51)$. Para el grupo de ideación suicida se utilizó una muestra no probabilística por conveniencia, seleccionando todos aquellos casos que fueron atendidos en el centro de adolescentes hasta diciembre de 2010 y se excluyeron todos aquellos que presentaron algún trastorno psicopatológico, retraso mental o adicción a sustancias. Para el grupo control se ajustó la muestra con base en el género y la edad del grupo de casos, el cual se formó por estudiantes de varias escuelas de Hermosillo utilizando como criterio básico de inclusión no haber presentado algún tipo de comportamiento suicida y como exclusión la adicción a sustancias adictivas. La media de edad fue de 15.51 años de edad, con una mínima de 12 y una máxima de 17. El género fue de una razón de 3:1 en relación femenino a masculino, siendo la media de años escolares cursados 9.69; en ambos grupos no se encontró diferencia en relación a las actividades rea-lizadas, el 94.2 por ciento de los adolescentes del grupo casos estudiaban y/o trabajaban y el 98 por ciento de los adolescentes del grupo control presentaban las mismas actividades. El grupo control presentó una mayor prevalencia de presencia del padre (92.4\%) que el grupo de casos (82.4\%); en relación a la presencia de la madre no hubo diferencia, el $72.5 \%$ del grupo de casos pertenecía a familias nucleares con ambos padres, con poca diferencia del grupo control (76.5\%).

\section{Instrumento de Medición}

El instrumento que se utilizó en esta fase fue estructurado con base en las variables que presentó el perfil psicosocial del adolescente atendido en el centro.

Para los factores sociales se utilizaron dos escalas: para las variables referentes a la percepción del joven en relación a la dinámica familiar, se utilizó la escala elaborada en México por Villatoro, Andrade-Palos, Fleiz, MedinaMora y Reyes (1997), la cual analiza el grado de percepción del adolescente en cuanto a la comunicación, apoyo y rechazo en relación con sus padres, los indicadores de confiabilidad reportan un Alfa de Cronbach de 0.70 para cada una de las áreas en población mexicana. Para medir los aspectos relacionados al maltrato infantil se utilizó la escala de tácticas de conflictos de Strauss, Hamby, Finkelhor, Moore y Runyan (1998), los reactivos miden la frecuencia de cómo los jóvenes percibieron alguna especie de maltrato de parte de sus padres en una escala de cero (Nunca) a seis (más de 20 veces en la semana), Strauss reportó un alfa de Cronbach de .79 en relación a la confiabilidad.

En relación a los factores psicológicos individuales se integraron las siguientes escalas: para la variable depresión se utilizó la escala del Centro de Estudios Epidemiológicos (CES-D) de Radloff (1977), en la versión adaptada para adolescentes (Roberts, 1980) se pide al estudiante que mencione el número de días que experimentó cada uno de los síntomas especificados durante la semana pasada. El formato de respuesta es tipo Likert, en donde "0" = ningún día de la semana pasada, "1" = a 1 ó 2 días, " 2 " = 3 ó 4 días y " 3 " = a entre 5 y 7 días de la semana anterior a la aplicación del cuestionario, de este modo, a mayor puntaje, mayor sintomatología depresiva; la escala a presentado un alto nivel de confiabilidad en adolescentes mexicanos (Alfa de .88) (GonzálezForteza, Ramos, Marino, \& Peña, 2001). 
Para la variable autoestima se incluyó la escala de Rosenberg (1965), la cual ha sido validada y revalidada para estudiantes mexicanos de secundaria y bachillerato por González-Forteza, Andrade y Jimémez (1997) presentando un alfa de .90 en relación a la confiabilidad.

En relación a las habilidades de afrontamiento y solución de problemas en los adolescentes se utilizó la escala de afrontamiento para adolescentes de Frydenberg y Lewis (1978). La escala es un inventario de auto-informe que permite evaluar confiabilidad de 18 estrategias diferentes y está compuesta de tres factores: resolución de problemas, ignorar el problema y afrontamiento improductivo. El formato de respuesta es tipo Likert, en donde "1" es igual a nunca lo hago, al "5" igual a lo hago con mucha frecuencia, la confiablidad presenta en Latinoamérica un alfa de .89.

Se utilizó también la segunda parte del inventario de Ansiedad Rasgo-Estado (Spielberg, Gorsuch, \& Lushene, 1982) la cual consiste en una escala tipo Likert que mide en términos generales el estado de ansiedad que presenta el adolescente con los puntaje "1 igual a casi nunca, "2" igual a algo, "3" igual a menudo, la confiabilidad quedó demostrada con un alfa de 0.70 en sus reactivos, aplicados a Latinoamérica. Para la variable impulsividad se utilizó la escala $F$ del cuestionario 16 PF de Cattel, Eber y Tatsuoka (1965), de los cuales se extrajeron cinco reactivos que mostraron en esta investigación una alta confiabilidad.

Con respecto a la variable dependiente, ésta consideró, por un lado, la ideación suicida, que se evaluó con cuatro reactivos diseñados por Roberts (1980), acerca del contenido cognoscitivo de pensamientos referidos a la muerte en general y de sí mismo. El intento suicida y las conductas auto - lesivas (parasuicidio) se midieron a través de las preguntas: "Alguna vez ¿te has herido, cortado, intoxicado?" y "¿Qué edad tenías la única o última vez que te hiciste daño a propósito?"; se han utilizado en estudios con población adolescente por González-Forteza, Marino, Rojas, Mondragón y Medina-Mora (1998). Además de las escalas anteriores, se utilizó un cuestionario de datos socio-demográficos para identificar a los participantes.

\section{Procedimiento}

Se recogió información socio-demográfica de la base de datos del centro de tratamiento de adolescentes y se localizaron a los adolescentes del grupo de casos en sus casas. Se les explicó la finalidad del estudio y se presentó una carta de consentimiento informado para solicitar la autorización de ellos y sus padres para aplicar el instrumento, la aplicación duró en promedio 30 minutos. Para la aplicación del grupo de control, se pidió permiso a directores de escuelas preparatorias y secundarias de Hermosillo, y se aplicó a los adolescentes en sus horas libres. Tras administrar los reactivos, se vació la información en una base de datos en SPSS, tras lo que se analizaron los resultados.

\section{Análisis Estadístico}

Los datos se analizaron a través del paquete estadístico SPSS 18 y EQS 6.1. Primeramente se obtuvieron estadísticas univariadas (medias y desviaciones estándar para las variables continuas y frecuencias para las variables categóricas). Posteriormente, se calcularon alfas de Cronbach para estimar la confiabilidad de las escalas utilizadas. También se calculó una matriz de correlaciones entre los promedios de las variables bajo estudio. Con el fin de agrupar las variables para conformar indicadores en el modelo estructural se elaboraron parcelas con los reactivos de las escalas. Se presume la representatividad de la parcela después de que se obtiene la consistencia de la escala, según lo indica el alfa de Cronbach.

Para elaborar el modelo estructural explicativo se especificaron dos factores de orden superior: Disfunción familiar (formado por los factores de primer orden "maltrato infantil", "comunicación familiar" y "rechazo de los padres") y Disfunción personal (formado por los constructos de "depresión", "ansiedad", "baja auto-estima" y "carencia de habilidades de afrontamiento"). Los factores de primer orden, a su vez, se conformaron por las parcelas previamente elaboradas, lo que aplicó también para los constructos de "ideación suicida" y "parasuicidio". De acuerdo con el modelo especificado, la disfunción familiar afecta a la disfunción personal, la cual, a su vez, influye en la ideación suicida y ésta repercute en el parasuicidio. 
El modelo fue puesto a prueba a través del programa EQS (Bentler, 2006) y la técnica de Modelamiento de Ecuaciones Estructurales (MEE). Para determinar si los datos de la investigación respaldan el modelo a prueba se consideraron los indicadores de bondad de ajuste. La comparación entre el modelo saturado (todas las interrelaciones entre las variables estudiadas) y el modelo teórico (que sólo considera las relaciones que el modelo considera relevantes) se llevó a cabo a través de dos tipos de indicadores: el indicador estadístico que requiere del empleo de la chi cuadrada (X2); y los indicadores prácticos: una serie de estadígrafos derivados de la X2 que controlan el efecto del número de sujetos sobre la significatividad de la comparación. En este caso se trata de probar que el modelo teórico no es diferente del saturado, por lo que se espera que el valor de la X2 sea bajo y no significativo; es decir, que su probabilidad asociada sea mayor a .05. Por otro lado, los indicadores prácticos requieren que sus valores se acerquen a 1 . El nivel mínimo para considerar el modelo con una adecuada bondad de ajuste es de .90. Los indicadores prácticos que se tomaron en cuenta fueron el Índice Bentler- Bonett de Ajuste No Normado (IANN) y el Índice de Ajuste Comparativo (IAC).

\section{Resultados}

La confiabilidad de los instrumentos utilizados se presenta en la Tabla 1. Las alfas de Cronbach fueron aceptables (Nieva \& Sorra, 2003), dado que su valor resultó entre .61, en el caso de la escala de parasuicidio, hasta .93, producido por la escala de auto-estima.

La matriz de correlaciones exhibida en la tabla 2 presenta la asociación encontrada entre el total de las variables del estudio. En ella es posible detectar asociaciones significativas entre las variables personales, así como entre las que constituyen el factor de disfunción familiar. También es posible captar que la ideación suicida y el parasuicidio se encuentran altamente correlacionados y que los factores personales, a la vez de relacionarse con los familiares, se asocian a la ideación suicida. Esta apreciación preliminar se ve confirmada, de manera sistemática en el modelo de ecuaciones estructurales.

La figura 1 muestra los resultados del modelo estructural. Primeramente se probó la validez del constructo de las variables latentes (disfunción familiar, disfunción personal, ideación suicida, parasuicidio, maltrato infantil, rechazo de los padres, déficit en la comunicación, depresión, ansiedad, baja autoestima y déficit en habilidades de afrontamiento). Este modelo produjo pesos factoriales altos y significativos en todos los indicadores y en los factores, los cuales indican validez convergente (Corral \& Figueredo, 1999). Las correlaciones altas y significativas entre los indicadores y su correspondiente factor fueron tomadas como evidencias de validez del constructo de los modelos de medición (Corral, Frías, \& González, 2001).

El modelo de ecuaciones estructurales revela que el origen de los comportamientos suicidas se encuentra en el factor de disfunción familiar, representado por el maltrato infantil, el rechazo de los padres y el déficit en la comunicación familiar. Este factor produce una relación directa con el constructo de disfunción individual, compuesto por las variables: ansiedad, depresión, baja autoestima y déficit en habilidades de afrontamiento. A su vez, la disfunción individual afecta de forma directa a la ideación suicida, la que influye en el parasuicidio.

Lo anterior sugiere que los problemas familiares afectan el comportamiento suicida en el adolescente al desencadenar una serie de trastornos y déficits en el mismo; éstos hacen más probable la planeación de actos suicidas; los que pueden materializarse como intentos de suicidio o, por lo menos, conductas autolesivas. La capacidad explicativa de este modelo es sobresaliente: la R2 de la ideación suicida es de .76 y la de parasuicido es de .79. Los indicadores prácticos de bondad de ajuste (reportados en el pie de la figura 1) señalan que los datos respaldan la pertinencia del modelo teórico de relaciones. 
Tabla 1

Medias e indicadores de confiabilidad de los instrumentos utilizados

\begin{tabular}{llll}
\hline Instrumento & $\mathrm{N}$ & Media & Alfa \\
\hline Rechazo Padre & 102 & 1.48 & .74 \\
Comunicación Familiar & 102 & 2.5 & .81 \\
Maltrato infantil & 102 & .532 & .87 \\
Autoestima & 102 & 2.85 & .93 \\
Ansiedad & 102 & 1.61 & .82 \\
Depresión & 102 & 1.79 & .86 \\
H. de Afrontamiento & 102 & 3.77 & .80 \\
Ideación Suicida & 102 & 1.47 & .90 \\
Parasuicidio & 102 & .39 & .61
\end{tabular}

Tabla 2

Matriz de correlaciones entre las escalas

\begin{tabular}{|c|c|c|c|c|c|c|c|c|c|}
\hline$\overline{V^{\prime} S}$ & AUT & ANS & DEP & $\mathrm{HA}$ & $\mathrm{RP}$ & CF & $\mathrm{MI}$ & IS & PS \\
\hline AUT & 1 & & & & & & & & \\
\hline ANS & -.12 & 1 & & & & & & & \\
\hline DEP & $-.26^{\star *}$ & $.46^{\star *}$ & 1 & & & & & & \\
\hline $\mathrm{HA}$ & $.23^{\star \star}$ & -.18 & $-.27^{\star *}$ & 1 & & & & & \\
\hline $\mathrm{RP}$ & $-.24^{*}$ & $.30^{\star *}$ & $.44^{\star *}$ & $-.50^{\star \star}$ & 1 & & & & \\
\hline CF & $.37^{*}$ & -.16 & $-.24^{\star}$ & $-.37^{* *}$ & $-.35^{\star *}$ & 1 & & & \\
\hline $\mathrm{MI}$ & $-.22^{\star}$ & $-22^{*}$ & $-26^{\star *}$ & -.18 & $.41^{* *}$ & $-.43^{\star \star}$ & 1 & & \\
\hline IS & $-.44^{\star *}$ & $.37^{* *}$ & $.39^{\star *}$ & $-.27^{\star *}$ & $.27^{\star \star}$ & $-.23^{*}$ & $-.23^{*}$ & 1 & \\
\hline PS & $-.24^{\star *}$ & $.45^{\star \star}$ & $.42^{\star \star}$ & $-.32^{\star \star}$ & $.21^{\star *}$ & -.17 & .19 & $-.58^{\star *}$ & 1 \\
\hline${ }^{*} p<.05$ & ${ }^{* *} p<.01$ & & & & & & & & \\
\hline
\end{tabular}




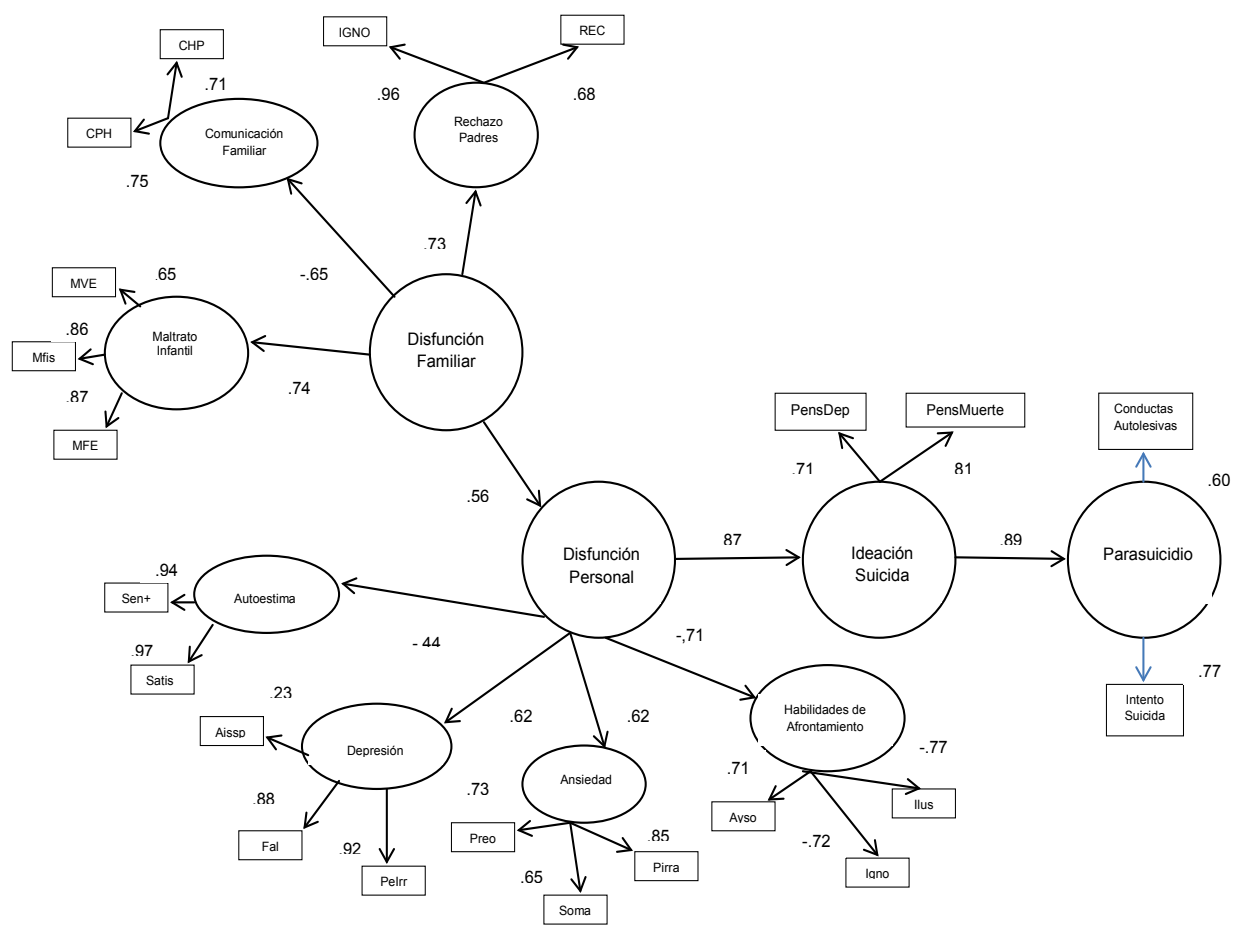

Figura 1. Modelo Estructural Explicativo del Comportamiento Suicida (Ideacion Suicida y Parasuicidio)

Bondad de Ajuste X2=283.21 (177 gl), $\mathrm{p}<.001 ; \mathrm{BNNFI}=.91 ; \mathrm{CFI}=.92 ; \quad \mathrm{RMSEA}=.07 ; \mathrm{R} 2$ Ideación Suicida $=-76$;

R2 Parasuicidio $=.79$

\section{Discusión}

Los resultados del presente estudio proporcionan una visión general de los orígenes de la conducta suicida en un grupo de adolescentes en la ciudad de Hermosillo, Sonora. Aunque no prestamos especial atención a factores situacionales de índole extra-familiar, sí consideramos algunos de ellos, como las actividades globales realizadas por los jóvenes (estudio, trabajo), las cuales no fueron determinantes en relación con la conducta suicida. Partiendo de lo que dice Fernández (2002), lo anterior representa que estas conductas pueden estar presentes independientes de la actividad que realizan los adolescentes de tal forma que no son factores de riesgo. Los dos grupos estudiados (incluyendo el que tenía antecedentes de esa conducta) pertenecen en su mayoría a escuelas de tiempo completo y algunos son trabajadores de medio tiempo.
Los resultados presentan una correlación entre todas las variables, tanto psicológicas como sociales, y nuestra variable dependiente, al igual que lo documenta la literatura revisada, en relación a la presencia de estos factores y su influencia con los comportamientos suicidas. Sin embargo, los resultados indican un continuo diferente de cómo se relacionan estas variables. Al comportamiento suicida frecuentemente se le asocia con tres problemáticas individuales importantes: el fracaso en la resolución de problemas interpersonales o falta de habilidades de afrontamiento, el alto nivel de desesperanza o indefensión aprendida (depresión), y una mala regulación del afecto o pobre control de impulsos (Sidley, 2001). De los anteriores quedó descartada la impulsividad, debido a que no se relacionó con ningún factor independiente ni con la variable dependiente.

El modelo de ecuaciones estructurales hace hincapié en los elementos 3, 4, 5, 6 y 8 del esquema que propone Mack (1986); lo que implica que, sin desestimar la influencia de factores culturales y biológicos en el suicidio, es posible explicar 
la mayor parte de este comportamiento como resultado de una disfunción psicológica (factores personales) y ésta, a su vez, es resultado fundamental de la disfunción familiar.

Coincidiendo con lo anterior se señala que la familia es un factor de gran importancia en la determinación del comportamiento suicida, coincidiendo con estudios anteriores a nivel internacional, los cuales muestran que en poblaciones donde los adolescente pertenecen a familias con una dinámica y estructura adecuada la prevalencia de estas conductas tienden a disminuir (Vougiouklakis, Tsiligianni, \& Vassilik, 2009). El factor de disfunción familiar influyó significativamente en trastornos y déficits de conducta individual (depresión, ansiedad, carencia de habilidades de afrontamiento, baja auto-estima), y éstos fueron poderosos determinantes de la ideación suicida. Como lo establece Mack (1986) en su modelo arquitectónico, las experiencias tempranas se presentan en la familia, y las habilidades de afrontamiento se aprenden $\mathrm{o}$ adquieren durante esta etapa; de ahí la importancia de la comunicación familiar y el efecto negativo del rechazo parental y el maltrato infantil (Rice, 2000).

Con lo anterior, se puede discutir hasta qué punto la dinámica familiar afecta los comportamientos suicidas. Sin embargo, este impacto no es directo como algunos estudios lo han afirmado (González-Forteza \& Jiménez. 2003; Compton et al. 2005), sino que se convierte en un factor de impacto directo para la generación de comportamientos que encajan en la falta de adaptación del desarrollo adolescente (Dulanto, 2000). La problemática psicológica resultante y la ausencia de factores protectores que ayuden al adolescente a solucionar sus problemas de manera asertiva y eficiente parecen conducir de inicio a la ideación suicida (Barcelata, Duran, \& Lucio, 2004; Palacios et al. 2007), no a las conductas parasuicidas.

En relación a la predictibilidad el hecho de que los primeros expliquen el $76 \%$ de la varianza de la segunda se constituye en una alerta, pero también en una información valiosa que puede aprovecharse para la prevención de las conductas auto-lesivas en los adolescentes. Un porcentaje tan elevado de capacidad explicativa indicaría que el trabajo de prevención debería enfocarse en el entorno familiar y en el empoderamiento de los adolescentes, en aras de fortalecer su autoestima, las habilidades de afrontamiento y su estado anímico.

Por lo anterior, se hace necesario ir más allá de la prevención en los aspectos individuales psicológicos del adolescente; no significa que no se trabaje con los adolescentes en habilidades para reducir los problemas de estrés, depresión, mejorar el auto-concepto o trabajar en la solución de problemas, lo cual es necesario hacerlo. Sin embargo, el enfocarnos solamente en lo anterior no reducirá la prevalencia del problema, si bien las anteriores tienen una relación directa con las conductas suicidas, no se pueden considerar como el inicio de las mismas, por lo que es necesario abordar el problema desde su génesis; el trabajo con las familias implicaría atacar las raíces de los sistemas de crianza castigantes (maltrato), mejorar los patrones de comunicación entre los miembros de la familia y procurar el establecimiento de relaciones afectuosas y de apoyo.

Muchos de esos procesos no son desplegados por falta de habilidades paternas (Strauss, Hamby, Finkelhor, Moore, \& Runyan, 1998), aunque no debe desconocerse que la violencia familiar posee raíces tan o más complicadas como las que generan la conducta suicida. Aun así, la falta de habilidades paternas incluye la ignorancia acerca de las consecuencias nocivas de la disfunción familiar. Ignorancia no equivale a despreocupación y es probable que muchos padres sean poco afectuosos e incluso agresivos con sus hijos porque suponen que esas son formas apropiadas de crianza (Frías \& McCloskey, 1998). También es probable que el conocimiento de que el maltrato, la falta de comunicación familiar y las manifestaciones de rechazo pueden conducir a los hijos a la ideación suicida -y de ahí a las conductas parasuicidaslleve a los padres a modificar sus patrones conductuales. Ésta es un área de intervención y estudio que está abierta.

Por lo tanto, el trabajo familiar preventivo de los problemas psicológicos en los adolescentes debe iniciar en las etapas tempranas del desarrollo del individuo además de la formación de una familia, ya que se tiene que dirigir el proceso de prevención a los eventos que originan la disfunción individual desde la infancia, y no en la etapa de la adolescencia cuando los trastornos y las incapacidades se encuentran firmemente establecidos. 


\section{Referencias}

Andrade P., Betancourt, O. D., \& Camacho Valladares. M. (2003). Ambiente Familiar de Adolescentes que han Intentado suicidarse. Revista de Psicología Social y Personalidad, $19,55-64$

Arlaes, N. L., Hernández, S. G., Álvarez, C. D., \& Cañizarez G.T. (1997).Conducta suicida factores de riesgo asociados. Revista Cubana de Medicina Integral, 14(2), 122-126.

Barcelata, B., Duran, C., \& Lucio, E. (2004). Indicadores de malestar psicológico en un grupo de adolescentes mexicanos. Revista Colombiana de Psicología. (13), 64-73.

Bentler, P. M. (2006). EQS 6 Structural Equations Program Manual. Encino, CA: Multivariate Software Inc.

Bertolote, J. M., \& Fleischmann, A. (2002). A global perspective in the epidemiology of suicide. Suicidologi, 7, 6-8.

Bobes, G. J. (2004). Comportamientos suicidas. Prevención y tratamiento. Barcelona: Ars Medica.

Castañeda, A. (2003). Características psicosociales del adolescente parasuicida. Recuperado de http://www.psicoactiva.com/ Noticias/n_ 0004

Casullo, M. M. (2002). Narraciones de adolescentes con alto riesgo suicida. Psicodiagnosticar, 12, 43-52

Cattel, R., Eber, H., \& Tatsuoka, M. (1965). Cuestionario de 16 factores de la personalidad. Editorial. Manual Moderno. México.

Compton, M., Thompson, N., \& Kaslow, N. J. (2005). Social environment factors associated with suicide attempt among low-income African Americans: The protective role of family relationships and social support. Soc Psychiatry Psychiatr Epidemiol. 40: 175-185.
Corral, V., \& Figueredo, A. J. (1999). Convergent and divergent validity of three measures of conservation behavior: The multitraitmultimethod approach. Enviroment \& Behavior, 31, 805-820.

Corral, V., Frías, M., \& González, D. (2001). Análisis cuantitativos de variables latentes. Hermosillo, México: UniSon.

Cubillas, M. J., Román, R., Abril, E., Terrones G., Lechuga, A., Martínez, Y., \& Moysen S. (2006). Ideación suicida e intento de suicidio en adolescentes sonorenses de educación media superior y su relación con la dinámica social y familiar. Reporte Técnico entregado a la Dirección General de Salud Mental de la Secretaría de Salud del Estado de Sonora.

Desjarlais. R., Eisenberg, L., Byron, G., \& Kleinman, A., (1995). World Mental Health. Problems and prioities in low-income countries. Reino Unido: Oxford University Presss.

Diekstra, R. (1985). Suicide and suicide attempts in the European Economic Community: an analysis of trends with special emphasis upon trends among the young. Suicide and LifeThreatening Behavior, 5, 27-42.

Dulanto, G. E. (2000). El adolescente. México: Mac Graw Hill Interamericana.

Durkheim, E. (1897). El Suicidio. México: Universidad Nacional Autónoma de México.

Dwairy, M. (2011). Perceived Family and School Rejection and Adolescents' Psychological States. Psychology, 2, 535-541.

Fernández, C. (2002). Factores asociados al intento de suicidio en la población colombiana. Revista Colombiana de Psiquiatría, 31, 283297.

Frías, M., \& McCloskey, L. A. (1998). Determinants of harsh parenting in Mexico. Journal of Anormal Child Phycology. 26, 129-139. 
Frydenberg, E., \& Lewis. (1978). ACS. Escala de afrontamiento para adolescentes. TEA Ediciones Madrid, España.

Garcia de Jalon, E., \& Peralta, V. (2002). Suicidio y riesgo de suicidio. Anales Sis San Navarra, 25 (Supl 3), 87-96.

González-Forteza, C., Andrade, P., \& Jiménez, A. (1997). Estresores cotidianos familiares, sintomatología depresiva e ideación suicida en adolescentes mexicanos. Acta Psiquiátrica y Psicológica de América Latina, 43, 319- 326.

González-Forteza C., Mariño M. C., Rojas E., Mondragón L., \& Medina-Mora M. E. (1998). Intento de suicidio en Estudiantes de la Ciudad de Pachuca, Hidalgo, y su relación con el Malestar Depresivo y el uso de Sustancias. Revista Mexicana de Psicología, 15, 165-175.

González-Forteza, C., Ramos, L., Mariño, M. C., \& Pérez, E. (2001). Vidas en Riesgo: Conducta suicida en adolescentes. Acta Psiquiátrica y Psicológica de América Latina, Buenos Aires, Argentina. En prensa.

González-Forteza, C., Villatoro, J. A., Alcántar, I., Medina-Mora, M. E., Fleiz, C., Bermúdez, P., \& Amador, N. (2002). Prevalencia de intento suicida en estudiantes de la Ciudad de México: Medición 2000. En AMEPSO (Eds.): La Psicología Social en México, vol. 9 (pp. 298-304).

González-Forteza, C., \& Jiménez, T. A. (2003). Veinticinco años de investigación sobre suicidio en la dirección de investigaciones epidemiológicas y psicosociales del instituto nacional de psiquiatría "Ramón de la Fuente". Salud mental. 26(6), 35-51

Guiao, I., \& Esparza, D. (1995).Suicidality correlates in MexicanAmericans. Issues in Mental Health Nursing, 16, 461-479.

Gunnell, D. J. (2002). The epidemiology of suicide. International Review of Psychiatry, 12, 21-26.

Gutiérrez, G. A., \& Contreras, C. (2006). El Suicidio conceptos actuales. Salud Mental. Sept-Oct. Año/Volumen (29), 66-74.
Hardt, J., Sidor, A., Nickel, R., Kappis, B., Petrak, P., \& Egle, U. T. (2008). Childhood adversities and suicide attempts: A retrospective study. Journal of Family Violence. (23), 713-718

Harris, T. E., \& Lennings, C. J. (1995). Suicide and Adolescence. International Journal of Offender Theraphy and Comparative Criminology, 37, 263-270

Huey, S., Henggeler, S., \& Rowland, M. (2004). Multisystemic therapy effects on attempted suicide by youths presenting psychiatric emergencies. Journal of the American Academy of Child and Adolescent Psychiatry, 4, 183-190.

Larraguibel, M., Martínez, V., Valenzuela, R., González, P., \& Schiattino, I. (2001). Intento de suicidio en adolescentes. Factores asociados. Psicopatología, 21, 237-253.

Leslie, M. B.; Stein, J. A. \& Rotheram-Borus, M. J. (2002). Sex specific predictors of suicidality among runaway youth. Journal of Clinical Child and Adolescents Psychology, 31, 27-40.

Mack, J. E. (1986) Adolescent suicide: aix arquitectural model. En G. L. Ríerman (ed.), Suicide and depression among adolescent and young adults. (pp. 53-76). Washington: American Psychiatryc Press.

Mardomingo, M. (1998). Los intentos de suicidio. En J. Rodríguez (Ed.), Psicopatología del niño y del adolescente $2^{a}$ ed (pp. 709-714). Tomo I. Sevilla: Universidad de Sevilla.

Marquet, B. (2008). Child and teenage suicide in Europe: a serious public health issue" Assemblée parlementaire. Strassburg.

Mittendorfer-Rutz, E., Wasserman, D., \& Rasmussen, F. (2008). Fetal and childhood growth and the risk of violent and non-violent suicide attempts: a cohort study of 318953 men. J Epidemiol Community Health. 62:168173 doi:10.1136/jech.2006.057133

Monge, H. A., Cubillas, R. M., Roman, P. R., \& Abril, V. E. (2007). Intentos de suicidio en adolescentes de educación media superior y su relación con la familia. Psicología y Salud, $17,45-51$. 
Montenegro, H., \& Guajardo, H. (2000). Psiquiatría del niño y del adolescente. 2da ed. Santiago, Chile: Editorial Mediterráneo.

Nieva, V. F., \& Sorra, J. (2003). Safety culture assessment: a tool for improving patient safety in healthcare organizations. Quality Safe Health Care, 12 (supl), ii17-ii23.

Nrugham, L., Larsson, B., \& Sund, A. M. (2008). Specific depressive symptoms and disorders as associates and predictors of suicidal act across adolescence. Journal of Affective Disorders. 111(1), 83-93.

Organización Mundial de la Salud (2009). Recuperado de:

http://www.who.int/mental_health/prevention/ suicide/wspd_2009_statement.pdf

Organización Panamericana de la Salud. (1998). La condición de salud en las Américas, Vol I: p.80. Washington, D.C. Recuperado de: http:// www. paho.org/spanish/HIA1998/SaludVol1. pdf

Palacios X., Barrera A. M., Ordoñez M., \& Peña M. A. (2007). Análisis bibliométrico de la producción científica sobre suicidio en niños en el período 1985-2005. Avances en Psicología Latinoamericana, 25, 40-62.

Radloff, L. S. (1977). The CES-D scale: a selfreport depression scale for research in the general population. Applied Psychological Measurement, 1, 385-401.

Reinherz, H., Tanner, J., Berger, S., Beardslee, W., \& Fitzmaurice, G. (2006). Adolescent Suicidal Ideation as Predictive of Psychopathology, Suicidal Behavior, and Compromised Functioning at Age 30. Am J Psychiatry, 163, 1226-1232.

Rice, F. (2000). Adolescencia: desarrollo, relaciones y cultura. Madrid: Prentice Hall.

Rivera, H. E., \& Andrade, P. (2006). Recursos individuales y familiares que protegen al adolescente del intento suicida. Revista intercontinental de Psicología y Educación. Año/Vol. 8. Numero 002
Roberts, E. (1980). Reliability of the CES-D Scale in Different Ethnic Contexts. Psychiatry Research 2,125-134.

Rosenberg, M. (1965). Society and the adolescent self-image. Princeton, N.Y.: Princeton University Press.

Ruiz, H. J., Navarro, J. M., Torrente H., \& Rodríguez González, A. (2005). Construcción de un cuestionario de creencias actitudinales sobre el comportamiento suicida. Psicothema, 17, 684-690.

Sauceda-García, J. M., Lara-Muñoz, M. C., \& Focil-Marquez, M. (2006). Violencia Autodirigida en la adolescencia: el intento de suicidio. Bol. Med. Hosp. Infant. Mex. Vol. 63, julio-agosto 2006.

Sean, J., Baser, R., Breeden, G., Neighbors, G., \& Jackson, J. (2006). Prevalence of and risk factors for lifetime suicide attempts among blacks in the United States. JAMA, 296, 21122123

Sidley, L. G. (2001). Parasuicide. En N. Tarrier, A. Wells \& G. Haddock (eds.), Treating Complex Cases. The Cognitive Behavioural Therapy Approach (pp. 272-275). Nueva York: Wiley.

Soo Kim, H., \& Sil Kim, H. (2008). Risk Factors for Suicide Attempts among Korean Adolescents. Child Psychiatry Hum Dev, 39, 221-235 DOI 10.1007/s10578-007-0083-4

Spielberg, Ch., Gorsuch, R., \& Lushene, R. (1982). Manual del Cuestionario de Ansiedad, Estado Rasgo ( $3^{\mathrm{a}}$ ed.). Madrid: TEA.

Stefulj, J., Büttner, A., Kubat, M., Zill, P., Balija, M., Eisenmenger, W., Bondy, B., \& Jernej, B., (2004). 5HT-2C receptor polymorphism in suicide victims association studies in German and Slavic populations. European Archives of Psychiatry and Clinical Neuroscience, 254, 224-227.

Steinhausen, H. C., \& Winkler, C. W. (2004). The impact of suicidal ideation in preadolescence, adolescence, and young adulthood on psychosocial functioning and psychopathology in young adulthood. Acta Psychiatrica Scandinavica, 110, 438-45. 
Strauss, M. A., Hamby, S. L., Finkelhor, D., Moore, D. W., \& Runyan, D. (1998). Identification of child maltreatment with the Parent-Child Conflict Tactics Scales: Development and psychometric data for a national sample of American parents. Child Abuse and Neglect, 22, 249-270.

Valero, A. (2008). En aumento, los índices de suicidio de niños y adolescentes en México. Boletín UNAM-DGCS-180. Ciudad Universitaria. Universidad Nacional Autónoma de México.

Villatoro, J., Andrade-Palos, P., Fleiz, C., MedinaMora, ME., \& Reyes, I. (1997). La relación padres-hijos: una escala para evaluar el ambiente familiar en adolescentes. Salud Mental, 20, 2:21-27.

Vougiouklakis, T., Tsiligianni, C., \& Vassiliki, A. (2009). Children, adolescents and young adults suicide data from Epirus, northwestern Greece. Boumba Forensic Sci Med Pathol 5(4), 269-73. 